\title{
Dynamic Software Architecture for Medical Domain Using Pop Counts
}

\author{
UMESH BANODHA \\ Department of Computer Applications \\ Samrat Ashok Technological Institute \\ Vidisha (M.P.), INDIA
}

\author{
KANAK SAXENA \\ Department of Computer Applications \\ Samrat Ashok Technological Institute \\ Vidisha (M.P.), INDIA
}

\begin{abstract}
Over the past few decades, the complexity of software for almost any era has increased significantly. The aim of this paper is to provide an approach which not only feasible but also decision-oriented in medical era. It focus on the careful planning and organizing success in continuous process improvements in software and hardware technology as this brings a lot of trouble to system development and maintenance. We have used the pop count method to develop the dynamic software architecture with the existence of quality attributes in order to find out the level of severity in patients of any diseases on the specialist perception. This is useful for taking decision on priority healing and regular concentration of the patients even in the absence of the specialist. Further the method (model) tested on the 25 symptoms of 100 patients which does not contain any dichotomous data and found with the help of statistical evaluation (that it result almost perfect classification) that the architecture is conformance to the medical software architecture quality requirements.
\end{abstract}

Keywords-Software Architecture; Quality Attributes; Pop count; medical process reengineering

\section{INTRODUCTION}

\section{A. Objective}

The medical era consists of two broadly classified data (1) Process Data and (2) Application data. Process data is actually the data which managed by medical system where as the application data is transformed across task units.

In fact, the great efforts is the implementation of good human factors practices in the design of software specially in the domain of medicine either is its concerned with the devices, diagnosis, usage, treatment etc. However, the survey finding indicates that lack of attention to human factors during software development may lead to errors that have the potential for patient's health or even death [11]. Thus, the design principles and participation of human factors especially in Indian scenario are very important, in order to increase the patients safety and authentication of the software in the medical era. Today, also the perception of specialists' doctors in remote areas is not up to the mark for the acceptability of the software in the decision / diagnosis and treatment.

For the above problem, Software Architecture is being widely used to describe a very high level design of large software systems. "The software Architecture of a program or computing system is the structure or structures of the system, which comprise software elements, the externally visible properties of those elements, and the relationships among them"[1]. Really it will be interesting to find out where software fits in with the software development life cycle especially in medical domain.

Architecture is the structure of the components of a program or system, their interrelationships and the principles and guidelines governing their design and evolution over time. [1]

The software architecture of a program or computer system is the structure or structures of the system, which comprise software components, the externally visible properties of those components, and the relationships among them. [2]

The importance of software architecture arose at very first step during system development especially in medical domain as they virtually affect every later stages of the development process which will direct or indirect impact the mortality rate of the patient at the end. Thus, good software architecture can reduce the risk with building a technical solution and make the system implementation and testing more traceable as well as achieve higher quality attributes. [3]

\section{B. Need of Software Architecture in Medical Domain}

Using software architecture we can present a common abstraction of a medical decision system that most if not all of the system's user can use as a basis for mutual understanding, negotiations, consensus and communication.

Medical architecture manifests the earliest design decisions about a system, and these early binding carry weights far out of proportion to their individual gravity with respect to the system's remaining development, its deployment and maintenance. The structure defines constraints on implementation. It explains the organizational structure and predicts the qualities of system. The architecture makes it easier to reason about and manage changes. It helps in evolutionary prototyping. The architecture enables more accurate cost and schedule estimates.

Software architecture constitutes a relatively small, intellectually graspable medical model for how a system is structured and how its elements work together, and this model is transferable across system. [1]

\section{Medical Process modeling approach}

Medical process is defined as the art of healing, i.e., a gradual process of medicine tending to cure. It is a method that 
helps to understand the actions, work flow, and tasks of an organization, and how the tasks are executed. The focus in process modeling is on the functional processes which are entities that start with an initial event and end with a result. A process has always an input and an output, input triggers the process and process results in an output $[5,6]$.

The process consists of four steps (Figure1) in the highest abstraction level. Process begins when the patient arrives to the reception of a hospital/clinic to meet doctor /staff as an initial event. It ends when the patient is discharged as a result. The actor of the processes is a doctor with support unless mentioned otherwise $[4,7]$. In this paper we focused on phase 1 and 2 of Process modeling approach i.e. Arrival/ First assessment of patient and planning the care $[8,9,10]$.

\section{Requirements of medical domain}

As we know that the medical system requires high performance then we can study the behavior of the elements (components) in time frame, the frequency and volume of inter-element communication. The medical model requires the modifiability then we can assign the responsibility to elements so appropriate change done in the whole system efficiently and abruptly.

The medical model need highly secure system; we need to provide protection of each elements and inter-element communication. We also need to provide the access power of each element so that authenticate user can observer the specialized elements of the system.

The model should provide the scalability. The model should provide localize use of resources so all patients are get benefited. It also concerned with higher capacity replacements. The model is combination of components and connector so it represents the incremental subsets of the system and manages inter-component usage. The model should be reusable i.e. each component or sub-components of the system be reusable. We restrict inter-element coupling so that we extract an element it does not come out with too many attachments to its current environment to be useful [1].

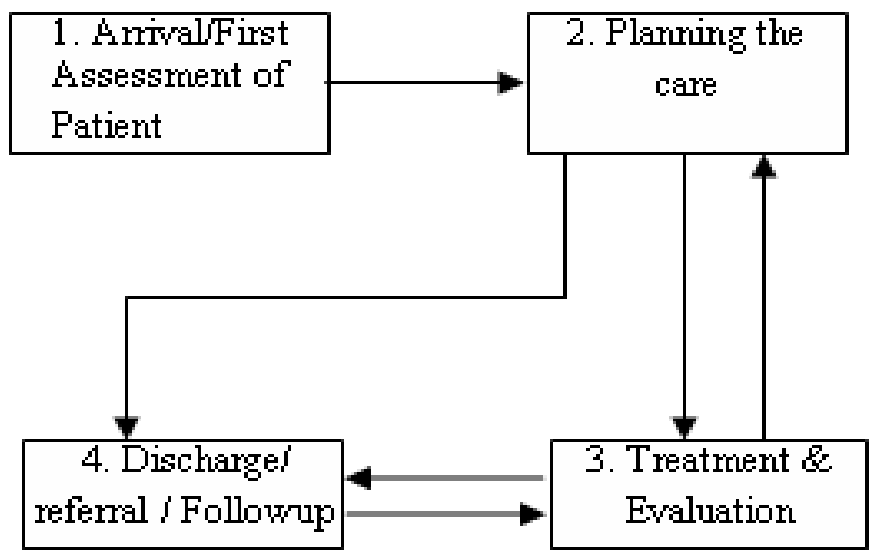

Fig. 1. Process Modeling Approach

\section{PROPOSED METHOD}

To make progress, we focus on the patient's symptoms caused by a specific set of medical conditions; and to generalize as the symptoms of patients of any diseases and the specialist will divide the set of their order. Thus, this paper gives the method which not only useful to the specialist but also a non-specialist i.e. moderate user can also use. For this we have work out on 100 patients' data with 25 attributes as symptoms and found the result in much improved manner.

Our method works on:

- We use the binary values as 0 if that symptom is not present otherwise 1.

- The medical specialist according to the diseases will decide a binary code of 25 values of 25 symptoms. These can be extended to as many as values required for the analysis.

- We proposed the dynamic software architecture using the pop count methods to estimate the level of severity among the set of the patients. Thus, in the absence of the specialist a moderate use can work out. The concept of this paper is to predict the level of severity with pop count method as the only sorting method cannot give the fruitful result

\section{A. Pop count Method}

We have the data of the patients in the form of symptoms sets which range from any number of symptoms to any number in any order except the data of patients should arrange in the same order $\mathrm{P}=\left\{\mathrm{P}_{1}, \mathrm{P}_{2}, \mathrm{P}_{3}, \ldots, \mathrm{P}_{\mathrm{n}}\right\}$ here $\mathrm{P}$ is the set of the patients,

$P_{i}=\left\{S_{1}, S_{2}, S_{3}, \ldots, S_{m}\right\}$ here $P_{i}$ is the set of individual patient's symptoms with $\mathrm{S}_{1}, \mathrm{~S}_{2}, \mathrm{~S}_{3}, \ldots, \mathrm{S}_{\mathrm{m}}$ symptoms.

Thus $\left\{P=S_{i j}\right.$ of all symptoms values of $i^{\text {th }}$ patients' $j^{\text {th }}$ symptoms $\}$

Now, in order to find out the patients' with critical condition we use the method of pop-count with count leading one's operation. We use the function as $\mathrm{f}\left(\mathrm{S}_{\mathrm{ij}}\right)=$ pop $\left(\mathrm{S}_{\mathrm{ij}} \wedge(\sim(-\right.$ $\left.\mathrm{S}_{\mathrm{ij}}\right)$ )) where ' $\wedge$ ' denotes the XOR bitwise operations. After performing the above function on the set $\mathrm{P}$ we indexed the entire data in order to find the list of critical data. Picking up any threshold value perform the preprocessing of the data and categories the data among various clusters in the styles (various types of styles) for various clusters depends upon the values ( standards) provided by the specialists' perception.

Figure 2 shown step by step activity of the pop-count method. We can observe that there are two decision steps by which we can dynamically observe the critical symptoms and take appropriate assessment.

\section{B. Software Architecture Evaluation}

The proposed software architecture will faster the treatment of serious patients. The method can be treated as the base for the software development which can have the following charactrestics. It can be of high performance, secure with less risk and due to its generality nature. It can be used for reusability. One level it gives the fast treatment with effectiveness on health point of view and on the other hand it may also affect medical spending. Though, we cannot do a complete evaluation of the impact of these changes, but we 
provide some information. In order to predict the level of severity in general we estimate logistic regression model as a function of severity on the presence of priority symptoms as

$$
F\left(P_{i}\right)=e^{\beta 0+\beta 1 s i j}
$$

i.e. the function of patients' equaling a "success" rather than a failure, where $\beta_{0}$ is the intercept and $\beta_{1}$ is the regression coefficient multiplied by the symptoms' success $\left(S_{i j}\right)$ and base e denotes the exponential function.

$$
\mathrm{L}\left(\beta_{0}, \beta_{1}\right)=\prod_{i=1}^{n} \mathrm{p}\left(\mathrm{s}_{\mathrm{ij}}\right)^{\mathrm{Ci}}\left(1-\mathrm{p}\left(\mathrm{s}_{\mathrm{ij}}\right)^{1-\mathrm{ci}}\right)
$$

On the basis of clusters on symptoms, we applied the weka software tool for predicting the assessment regarding patients' symptoms. The evaluation of model is done on kappa statics, mean absolute error, root mean square error, root Absolute error and root relative square error.

1) The Kappa Statistic: Interobserver variation can be measured in any situation in which two or more independent observers are evaluating the same thing. The calculation is based on the difference between how much agreement is actually present ("observed" agreement) compared to how much agreement would be expected to be present by chance alone ("expected" agreement).

2) Mean absolute error (MAE): The MAE measures the average magnitude of the errors in a set of forecasts, without considering their direction. The mean absolute error is a quantity used to measure how close forecasts or predictions are to the eventual outcomes

3) Root mean squared error (RMSE): The difference between forecast and corresponding observed values are each squared and then averaged over the sample. The RMSE is most useful when large errors are particularly undesirable. They are negatively-oriented scores: Lower values are better.

4) Relative Absolute Error (RAE): The relative absolute error is very similar to the relative squared error in the sense that it is also relative to a simple predictor, which is just the average of the actual values.

5) Root Relative Absolute Error (RAE): By taking the square root of the relative squared error one reduces the error to the same dimensions as the quantity being predicted.

\section{ANALYSIS}

The evaluation result of the proposed modal worked out on the 100 patients with 25 symptoms of any diseases. The result as per table 1 shows a perfect performance on the training data set and correctly classified all the data especially of class A, B that is no false negative and false positive in the report. The analysis reported as kappa with 1 i.e. perfect agreement in class A, B and some extent class C is also almost perfect where as in class D \& F it is substantial and class $\mathrm{E}$ an having moderate. Alternatively, we have used the relative errors to strengthen the result.

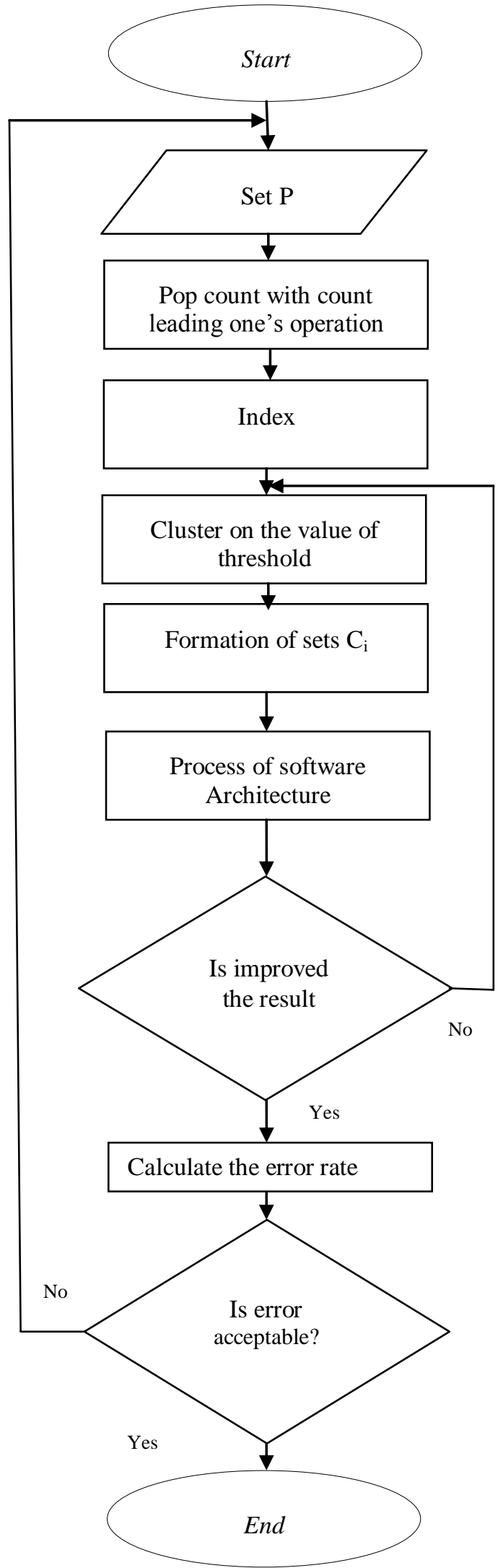

Fig. 2. Flow chart of method using pop-count 
It is discovered that in class $\mathrm{A}$ and $\mathrm{B}$ equal to zero and rest it range from 0.01 to 0.05 . In order to strengthen the software architecture evaluation we used MAE which ranges from 0.0 to 0.03 and other error rates. Thus, a model has a lower error rate will be preferred as it has more powerful classes capability and ability in terms of medical and bio-informatics fields. Statistical analysis shows that classes A and B are the perfect. The graphs are the pictorial representation of the various results.

Our proposed model is capable for effectively handle the patients data both on architectural and non-architectural aspect. The resultant is of high performance, even if the specialists change the order of symptoms and hence reduce the ambiguity and confusion in terms of risk. Thus, we can say that the model is having the quality attributes which are required in the architectural evaluation process maintainability, usability, performance, reliability, reusability and availability.

TABLE I. STATISTICS OF VARIOUS Clusters

\begin{tabular}{|l|r|r|r|r|r|r|}
\hline & F & A & B & C & D & \multicolumn{1}{|l|}{ E } \\
\hline Kappa & 0.89 & 1 & 1 & 0.95 & 0.84 & 0.72 \\
\hline MAE & 0.02 & 0 & 0 & 0.00 & 0.012 & 0.03 \\
\hline RMSE & 0.03 & 0 & 0 & 0.05 & 0.078 & 0.12 \\
\hline RAE & 10.10 & 0.00 & 0 & 5.02 & 16.00 & 27.90 \\
\hline RRSE & 31.78 & 0.03 & 0.01 & 22.41 & 40 & 52.84 \\
\hline
\end{tabular}

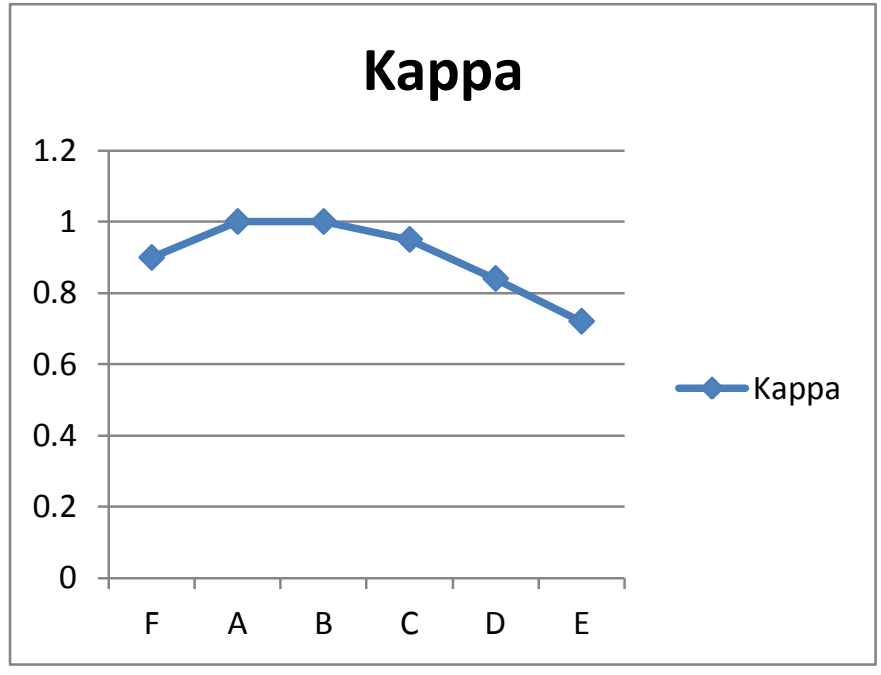

Graph I Statistics between clusters and kappa

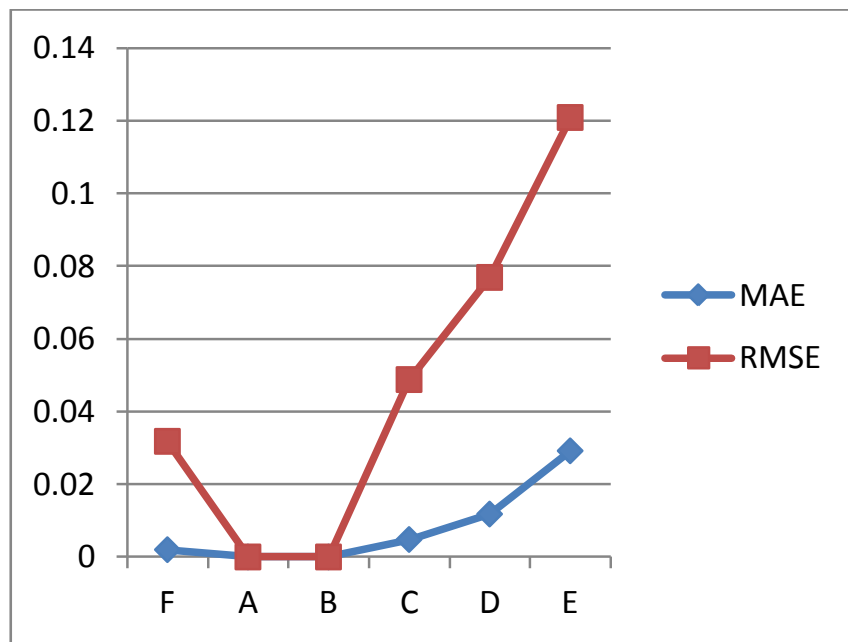

Graph II Statistics between clusters, MAE and RMSE

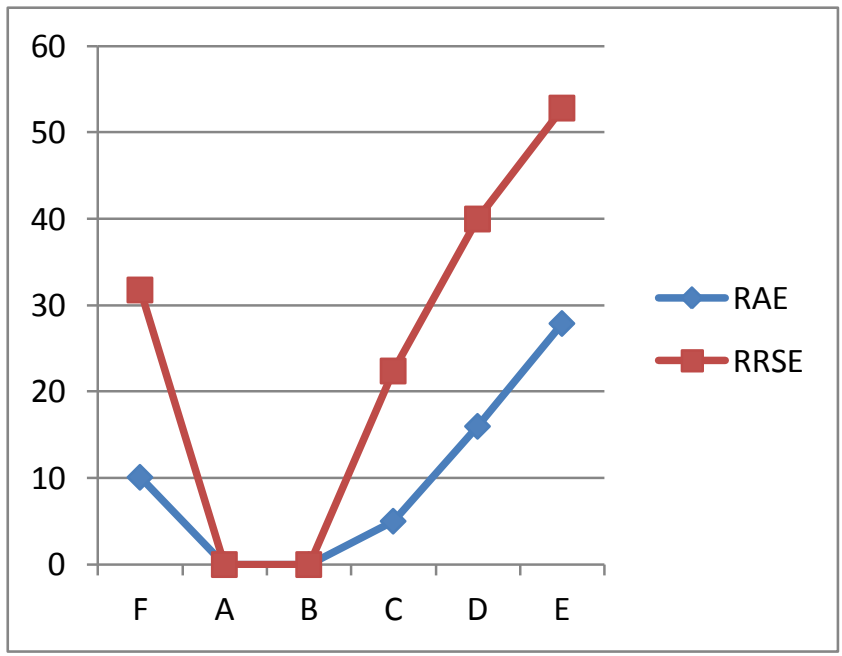

Graph III Statistics between clusters, RAE and RRSE

\section{CONCLUSIONS}

The paper deals with the analysis of model with the impact of software architecture based on 100 records (data sets) of general patients. As a conclusion, we have proposed a model which is to evaluate and investigate the level of severity of patients based on the pop-count. This will help not only the specialist but also the dichotomous and moderate users to decide about the useless information; useful information about a supposed illness on the evidence suggests that improvements in medical care with higher use experienced considerable reductions in mortality, even with the existence of human factors. The proposed software architecture uses all the patients with equal priorities and symptoms of which the order of their availability and non-availability are to be set by the specialist. This also use the quality attributes among them is of interdependencies. 


\section{REFERENCES}

[1] Len Bass,Paul Clements, Rick Kazman, "Software Architecture in Practice", Pearson education, Second edition", 2005.

[2] Banani Roy and T.C. Nicholas Graham, "Method for Evaluating Software Architecture: A Survey”, Technical Report 2008-545, School of computing queen's university at Kingston Ontario, Canada, April, 2008.

[3] Qiushi Wang, Zhao Yang, "A Method of selecting appropriate software architecture style/pattern: Quality Attributes \& Analytic Hiererchy Process", University of Gothenburg, Chalmers University of Technology, Göteborg, Sweden, June 2012

[4] P. Nykanen and J. Makinem, "Integration of medication information in electronics patient record systems", The dementia patient case. Turku School of economics, Research report LTH-1:2007, Turku, 2007.

[5] Wang, "Modelling information architecture for the organization. Information and Management" 32, 6, 1997, pp. 303-315.

[6] R. Weber, "Conceptual modeling and ontology: Possibilities and pitfalls", Journal of Database Management,14, 2, 2003, pp. 1-20.

[7] Makinen,J. Et.Al., "Process Models of medication Information", Procd. Of the $42^{\text {nd }}$ Hawaii International Conf. on System sciences 2009, 1-7.

[8] U.Banodha , K.saxena, "Impact of Pipe and Filter Style on Medical Process Re-engineering”, International Journal of Engineering Sciences, October 2011.

[9] U.Banodha , K.saxena,'Usability of Software Architecture design pattern in Medical process reengineering model", International Journal of Application or Innovation in Engineering \& Management (IJAIEM), Volume 2, Issue 6, June 2013, ISSN 2319 - 4847.
[10] U.Banodha, K.Saxena, "Comparison of Software Architecture Styles in Medical Process Re-engineering Model", International Journal of Wisdom Based Computing, Vol. 2(1), April 2012.

[11] http://www.ncbi.nlm.nih.gov/pubmed/15306163

\section{AUTHORS PROFILE}

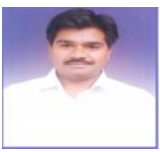

UMESH BANODHA, Assistant Professor at Samrat Ashok Technological Institute, VIDISHA (M.P.), an Autonomous Institute, affiliated to Rajiv Gandhi Technical University, Bhopal. I did MCA, M.Tech (Honors) and Pursuing Ph.D. My Area of interest Software Engineering / Architecture, Databases, UML, object oriented, Programming Languages etc. I am member of various international / National journals. I published more than 12 research papers in various conferences and journals (National / International).

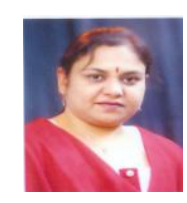

KANAK SAXENA, $\mathrm{Ph}$. D. in computer Science from the Devi Ahilya University, Indore, INDIA. She is professor in the Computer Applications Department at the Samrat Ashok Technological Institute affiliated to Rajiv Gandhi Technical University, Bhopal. Her Current research focuses on Database Systems, Parallel computing, Data Uncertainty and design and other interests include Network security and performance and Software Engineering. She is the member of editorial board of various international journals. She is the member of the international committee of the International Conference on Computer Science and Its Applications. She Published more than 80 research Papers in Various Conferences and Journals National / International). 